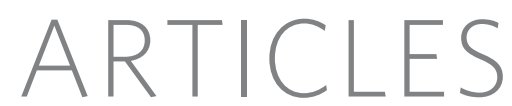

\title{
Mesoangioblast stem cells ameliorate muscle function in dystrophic dogs
}

\author{
Maurilio Sampaolesi ${ }^{1,2 \star}$, Stephane Blot ${ }^{3 \star}$, Giuseppe D'Antona ${ }^{2}$, Nicolas Granger ${ }^{3}$, Rossana Tonlorenzi ${ }^{1}$, \\ Anna Innocenzi ${ }^{1}$, Paolo Mognol ${ }^{4}$, Jean-Laurent Thibaud ${ }^{3}$, Beatriz G. Galvez ${ }^{1}$, Ines Barthélémy ${ }^{3}$, Laura Perani ${ }^{1}$, \\ Sara Mantero ${ }^{4}$, Maria Guttinger ${ }^{5}$, Orietta Pansarasa ${ }^{2}$, Chiara Rinaldi $^{2}$, M. Gabriella Cusella De Angelis ${ }^{2}$, \\ Yvan Torrente $^{6}$, Claudio Bordignon ${ }^{1}$, Roberto Bottinelli ${ }^{2} \&$ Giulio Cossu ${ }^{1,5,7}$
}

Duchenne muscular dystrophy remains an untreatable genetic disease that severely limits motility and life expectancy in affected children. The only animal model specifically reproducing the alterations in the dystrophin gene and the full spectrum of human pathology is the golden retriever dog model. Affected animals present a single mutation in intron 6 , resulting in complete absence of the dystrophin protein, and early and severe muscle degeneration with nearly complete loss of motility and walking ability. Death usually occurs at about 1 year of age as a result of failure of respiratory muscles. Here we report that intra-arterial delivery of wild-type canine mesoangioblasts (vessel-associated stem cells) results in an extensive recovery of dystrophin expression, normal muscle morphology and function (confirmed by measurement of contraction force on single fibres). The outcome is a remarkable clinical amelioration and preservation of active motility. These data qualify mesoangioblasts as candidates for future stem cell therapy for Duchenne patients.

Duchenne muscular dystrophy primarily affects skeletal muscle, causing fibre degeneration, progressive paralysis and death ${ }^{1}$. No effective treatment exists although novel therapeutic strategies, ranging from new drugs to gene and cell therapy, hold promise for significant advance in the future ${ }^{2}$. In particular, different types of stem cell have been shown to induce dystrophin synthesis and partial rescue of the pathology in dystrophic mice ${ }^{3-8}$. However, dystrophic mice do not display clinical signs of the disease, and to proceed to a clinical trial it is imperative to show efficacy in a large, non-syngeneic animal model of muscular dystrophy. Golden retriever muscular dystrophy (GRMD) $)^{9,10}$ is a very severe form of dystrophy, which affects not only limb, respiratory and heart muscles but also pharyngeal muscles, resulting in a severe involvement of the digestive tract; although variability exists between individuals, by 8 months of age most dogs walk with great difficulty (Supplementary Movie 1). To test the efficacy of cell or gene therapy, we transplanted GRMD dogs with either autologous genetically corrected or donor wild-type mesoangioblasts, under different regimes of immune suppression.

Ten dystrophic dogs were treated in three experiments and a general scheme of treatments and outcome is reported in Table 1. Four dogs received autologous mesoangioblasts, transduced in vitro with a lentiviral vector expressing human microdystrophin (Supplementary

Table 1 | Summary of treatment

\begin{tabular}{|c|c|c|c|c|c|c|c|c|}
\hline $\begin{array}{l}\text { Dog } \\
\text { no. }\end{array}$ & $\begin{array}{c}\text { Dog } \\
\text { name }\end{array}$ & Cell treatment & Lentiviral vector & $\begin{array}{l}\text { Onset of } \\
\text { treatment }\end{array}$ & $\begin{array}{l}\text { Immune suppression } \\
\text { (time) }\end{array}$ & $\begin{array}{l}\text { Dystrophin } \\
\text { expression }\end{array}$ & Motility & $\begin{array}{l}\text { Outcome of experiment } \\
\text { (at time P400) }\end{array}$ \\
\hline 01A & Ucal & Autologous, gene therapy & CK- $\mu$ dys-ires-GFP & P118 & - & $+/-$ & Loss & Euthanasia (P272) \\
\hline $02 \mathrm{H}$ & Vrillie & Heterologous, WT donor & - & P80 & CYC A (P78) & + & Loss & Euthanasia (P235) \\
\hline $\mathrm{O3H}$ & Valgus & Heterologous, WT donor & - & P75 & CYC A (P73) & +++ & No decline & Alive and well \\
\hline $\mathrm{O} 4 \mathrm{H}$ & Varus & Heterologous, WT donor & - & P75 & RAP (P73) & +++ & Modest decline & Alive and well \\
\hline $05 \mathrm{H}$ & Viko & Heterologous, WT donor & - & P77 & RAP + IL-10 (P74) & ND & ND (sudden death) & Myocarditis (P186) \\
\hline 06A & Vaccin & Autologous, gene therapy & MLC1F- $\mu$ dys & P113 & - & ++ & Major decline & Euthanasia (P326) \\
\hline 07A & Valium & Autologous, gene therapy & MLC1F- $\mu$ dys & P113 & - & ND & Loss & Pneumonia (P245) \\
\hline 08A & Vampire & Autologous, gene therapy & MLC1F- $\mu$ dys & P113 & - & ++ & Major decline & Pneumonia (P154) \\
\hline $09 \mathrm{H}$ & Azur & Heterologous, WT donor & - & P159 & CYC A (P157) & ++ & Restored & Alive and well \\
\hline $10 \mathrm{H}$ & Azor & Heterologous, WT donor & - & P159 & CYC A (P157) & +++ & Restored & Alive and well \\
\hline $11 U$ & Akan & None & - & - & - & - & Loss & Euthanasia (P380) \\
\hline $12 U$ & Vulcano & None & - & - & - & - & Loss & Euthanasia (P376) \\
\hline $13 U$ & Viking & None & - & - & - & - & Loss & Euthanasia (P340) \\
\hline
\end{tabular}

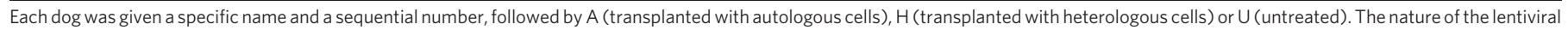

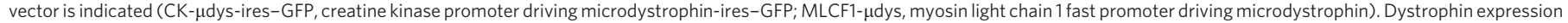

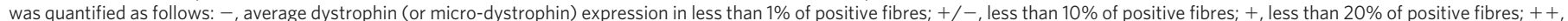

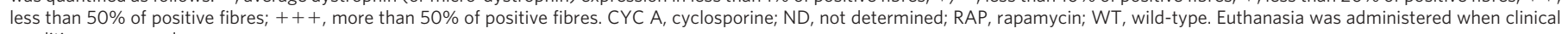
conditions worsened.

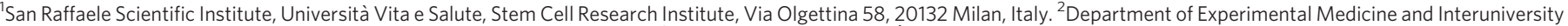

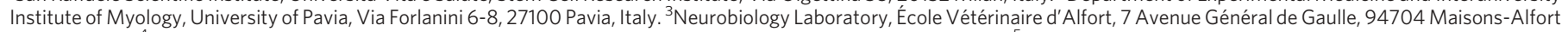

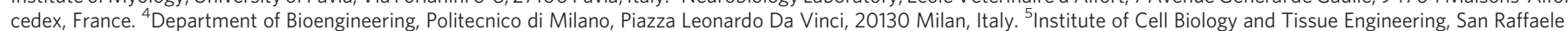

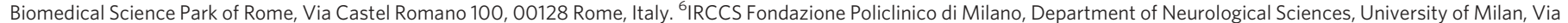
Sforza 35, 20122 Milan, Italy. ${ }^{7}$ Department of Biology and Centre for Stem Cell Research, University of Milan, Via Celoria 28, 20130 Milan, Italy.

*These authors contributed equally to this work. 
Fig. 1), a truncated but functional version of dystrophin ${ }^{11}$; six dogs received wild-type mesoangioblasts from a single DLA (dog leukocyte antigen)-unrelated donor under treatment with either cyclosporine or rapamycin. Three other dystrophic dogs were not treated and served as controls. All dystrophic dogs received steroids daily as standard treatment. Overall, the results showed that donor wild-type mesoangioblasts significantly ameliorate many symptoms of canine muscular dystrophy, whereas autologous genetically corrected cells are much less effective.

\section{Isolation and characterization of canine mesoangioblasts}

Both wild-type and dystrophic mesoangioblasts were isolated from the outgrowth of small, vessel-containing, tissue fragments from muscle biopsies performed after diagnosis, at about 15 days postnatal (P15). The cells show a morphology very similar to that of mouse mesoangioblasts $^{12}$ (Fig. 1a), proliferate efficiently in a medium devised for stem cells (Fig. 1b) and show a euploid kariotype of 78 chromosomes both at early and late passage (Fig. 1c); cells undergo senescence after about 25 population doublings. Canine mesoangioblasts express CD44 and CD13 but not CD34, CD45, CD117 or CD31 (not shown). Dog mesoangioblasts differentiate into multinucleated myotubes when co-cultured with $\mathrm{C} 2 \mathrm{C} 12$ mouse myoblasts or when transfected with MyoD. For the gene transfer experiments, proliferating mesoangioblasts isolated from dystrophic dogs were transduced with lentiviral vectors expressing human microdystrophin and (only for Ucal, the first dog treated) enhanced green fluorescent protein (EGFP). Both proteins became readily detectable after myogenic differentiation (Fig. 1d-f). Finally, to test the ability of these cells to reconstitute muscle fibres in vivo, both wild-type and GRMD genetically corrected mesoangioblasts were injected into SCID (severe combined immunodeficiency)-mdx mice, which do not reject xenogenic cells; the cells migrated from the femoral artery to the downstream muscles with an efficiency similar to that of their wild-type mouse counterparts ${ }^{13}$ (Fig. $1 \mathrm{~g}$ ). Three weeks after injection, canine mesoangioblasts gave rise to dystrophin-positive fibres containing dog nuclei, identified by anti-human lamin A-C antibody, which recognizes human and dog but not mouse nuclei (Fig. 1h, i). Thus, dog mesoangioblasts seem similar to their mouse postnatal counterparts by all the parameters tested, with the notable exception of a finite lifespan, a predictable difference between cells from rodents and other mammals.

\section{Feasibility experiment}

Dogs are identified by name and also by a sequential number followed by a letter (A for autologous cell transplantation, $\mathrm{H}$ for heterologous cell transplantation, and $U$ for untreated) (Table 1).

Two dogs, Ucal and Vrillie, were treated with three consecutive (at 1 -month intervals) injections of $5 \times 10^{7}$ cells into the femoral artery. Ucal (01A) received autologous cells, transduced with the lentiviral vector expressing human microdystrophin (Supplementary Fig. 1a). Vrillie $(02 \mathrm{H})$ received wild-type donor cells under a regimen of cyclosporine treatment.

During and after the treatment, Ucal $(01 \mathrm{~A})$ and Vrillie $(02 \mathrm{H}) \mathrm{did}$ not show appreciable sign of clinical amelioration and underwent a progressive decline in their walking ability. Biopsies, taken 1 month after the third injection, revealed variable morphology in different muscles of the injected legs, varying from severe and advanced degeneration in the tibialis cranialis of Ucal (01A), shown in Fig. 2a, to an intermediate severity in the same muscle of Vrillie $(02 \mathrm{H})$, shown in Fig. 2b. In general, the morphology of Vrillie $(02 \mathrm{H})$ was better but still variable, with several areas being quite well preserved. Dystrophin expression also showed variability and was in general correlated with morphology. At 8 months of age, the proportion of revertant fibres in dystrophic dogs varies from $0.02 \%$ to $0.3 \%$ (ref. 14). In the biopsies collected, dystrophin-positive fibres ranged from $2 \%$ to $7 \%$ in Ucal (01A) and from $4 \%$ to $10 \%$ in Vrillie $(02 \mathrm{H})$ (not shown). Figure $3 a, a^{\prime}$ shows a biopsy of Ucal in which clusters of dystrophin-positive fibres with several centrally located nuclei can be observed. Biopsies from contralateral, non-injected legs showed poorer morphology and a smaller proportion ( $2 \%$ or less) of dystrophin fibres. Unexpectedly, we found areas of dystrophin expression in the triceps brachialis of Vrillie $(02 \mathrm{H})$ (not shown), indicating that

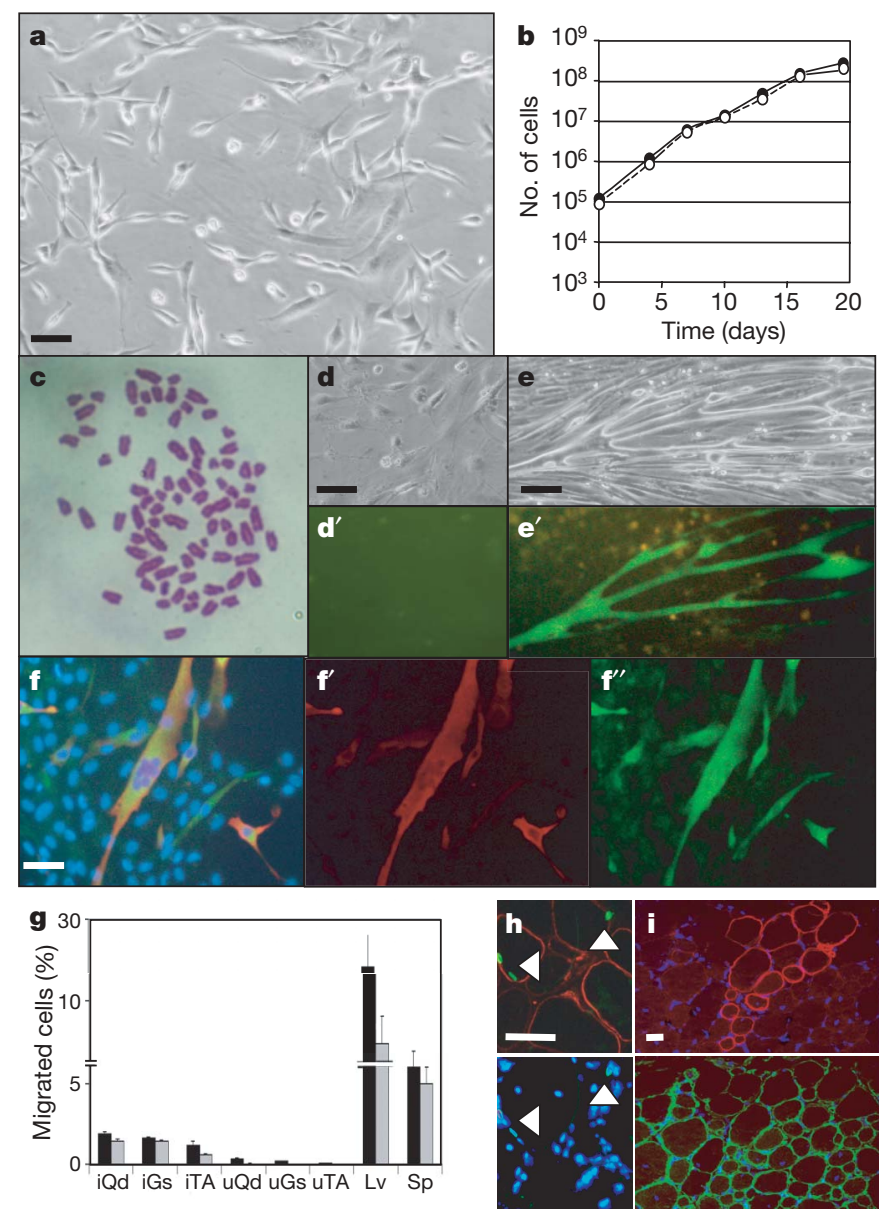

Figure 1 | Characterization of dog mesoangioblasts. a, Morphology of canine mesoangioblasts isolated from muscle biopsies of a golden retriever dog at P15. b, Proliferation curves of wild-type (filled circles) and dystrophic (open circles) canine mesoangioblasts. c, Karyotype of canine mesoangioblasts, which are consistently euploid until senescence. d-f, Transduction of dystrophic canine mesoangioblasts with a lentiviral vector expressing human microdystrophin and the EGFP gene reporter under the control of muscle-specific creatine kinase promoter. GFP expression is undetectable in proliferating mesoangioblasts $\left(\mathbf{d}, \mathbf{d}^{\prime}\right)$ but is readily detected in myotubes derived from the fusion of transduced mesoangioblasts with $\mathrm{C} 2 \mathrm{C} 12$ myoblasts $\left(\mathbf{e}, \mathbf{e}^{\prime}\right)$. Similar results were obtained after MyoD-induced differentiation: GFP-positive cells ( $\left.\mathbf{f}^{\prime \prime}\right)$ also express MyHC ( $\left.\mathbf{f}^{\prime}\right)$ as confirmed in the merged image (f), which also shows multinucleation. $\mathrm{g}$, Migration of canine mesoangioblasts into skeletal muscle: $5 \times 10^{5}$ mouse (black bars) or dog (grey bars) mesoangioblasts, previously transduced with a GFP-expressing lentiviral vector ${ }^{4}$, were injected into the right femoral artery of SCID-mdx mice. Six hours after injection, several muscles were isolated and the presence of donor cells was measured by real-time PCR analysis for GFP as detailed elsewhere ${ }^{13}$. Qd, quadriceps; Gs, gastrocnemius; Tc, tibialis cranialis, Lv, liver; Sp, spleen; the letter $\mathrm{i}$ (injected) before the muscle name indicates muscle isolated from the injected leg; the letter $\mathbf{u}$ (uninjected) indicates muscles isolated from the contralateral leg. $\mathbf{h}$, Top: immunofluorescence with antibodies against human lamin A-C (green) and dystrophin (red), revealing dog mesoangioblasts inside the muscle fibres of SCID-mdx mice, 21 days after intra-arterial injection. Bottom: nuclei were stained with 4,6-diamidino-2phenylindole (DAPI). i, Immunofluorescence of fibres with antibodies against dystrophin (red, top) and laminin (green, bottom) in the muscle of SCID-mdx mice, 21 days after intra-arterial injection. Scale bar, $20 \mu \mathrm{m}$. Error bars, 1 s.d. 
injected cells must to a certain extent transit through the capillaries of the injected leg and then through the filter organs, finally entering the arterial circulation to reach the forelimb muscles. Overall, the intensity of staining was weak; indeed, western blot analysis confirmed very low, barely detectable, levels of microdystrophin and dystrophin, respectively. Immunohistochemistry detected several inflammatory infiltrates containing mainly macrophages and lymphocytes (Supplementary Fig. 2a, b). Although these data demonstrate donor-celldependent dystrophin expression in dystrophic dogs and thus the feasibility of this therapeutic approach, the results were modest overall. To improve the efficacy of the treatment, the number of injections was increased to five (always at 1-month intervals), a new lentivector was produced in which muscle-specific creatine kinase was replaced by the stronger myosin light chain $1 \mathrm{~F}$ promoter and GFP was deleted because we could not detect GFP in unfixed cryostat sections (Supplementary Fig. 1b).

\section{Efficacy of heterologous wild-type mesoangioblasts}

Three dogs, namely Valgus, Varus and Viko $(03 \mathrm{H}, 04 \mathrm{H}$ and $05 \mathrm{H}$, respectively), were treated with donor cells. One of these dogs (Valgus, $03 \mathrm{H})$ received five arterial systemic injections $\left(5 \times 10^{7}\right.$ cells each) through a catheter that was introduced in the left femoralis artery and reached the aortic arch at the level of the left subclavia: cells were released mainly in the two large arteries. From a clinical point of view, Valgus $(03 \mathrm{H})$ had optimal performance and was still walking well 5 months after the last injection and the termination of immune suppression, at the age of 13 months (Supplementary Movie 2). Valgus $(03 \mathrm{H})$ was treated with cyclosporine, whereas the other two dogs, Varus $(04 \mathrm{H})$ and Viko $(05 \mathrm{H})$, were treated with rapamycin and with rapamycin and interleukin (IL)-10, respectively. Different protocols of immune suppression were tested to evaluate efficacy versus toxicity in this model, but the results did not show significant differences between cyclosporine and rapamycin. In fact, Varus $(04 \mathrm{H}$; Supplementary Movie 3) and Viko $(05 \mathrm{H})$ also had good clinical performance but after 2 months of treatment Viko $(05 \mathrm{H})$ died suddenly of a fulminans myocarditis whose cause remained unexplained. Varus $(04 \mathrm{H})$, in contrast, progressively lost walking ability after the
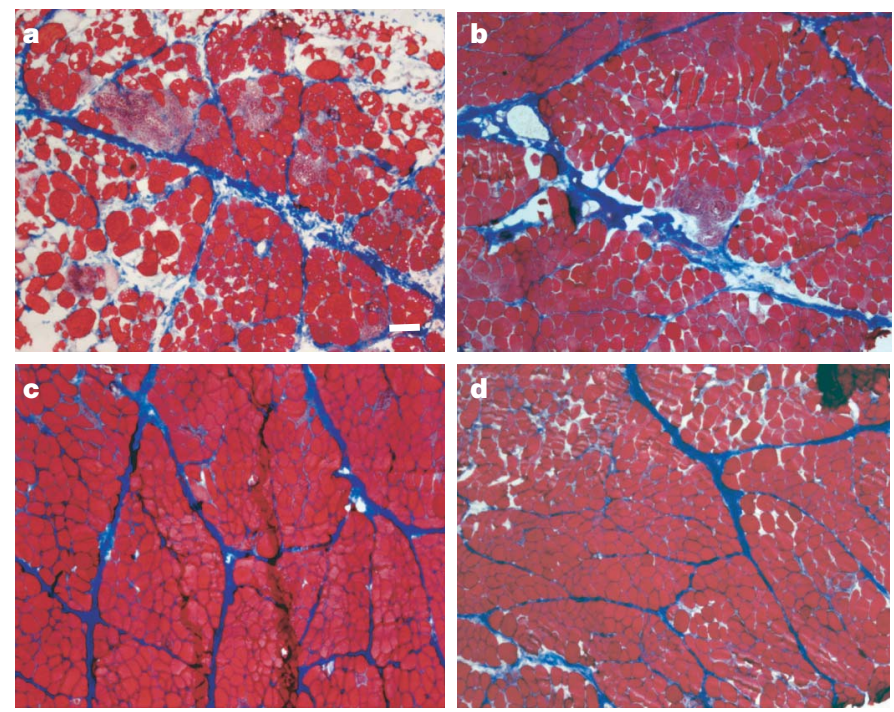

Figure 2 | Morphology of muscle in treated dogs. Azan Mallory staining of several muscle biopsies from the tibialis cranialis of dogs transplanted with heterologous mesoangioblasts or autologous, genetically corrected, mesoangioblasts. a-c, Examples show variability from severely affected tissue of Ucal $(01 \mathrm{~A} ; \mathbf{a})$ and Vrillie $(02 \mathrm{H} ; \mathbf{b})$, with many infiltrates, collagen and fat deposition, to the almost normal appearance of tissue from Valgus $(03 \mathrm{H} ; \mathrm{c})$, showing only thickened interstitial tissue. $d$, A biopsy from Vaccin (06A) shows an intermediate situation with well-preserved morphology. Bar $=100 \mu \mathrm{m}$. end of immune suppression. Unexpectedly, however, this animal had no detectable anti-dystrophin antibodies and his circulating lymphocytes did not react to donor mesoangioblasts or to protein extracts from the transplanted muscle (Supplementary Fig. 1c, d).

At the end of the treatment, biopsies were taken from several muscles of the injected and contralateral leg of these dogs, treated with heterologous wild-type cells. Histological analysis of biopsies from Valgus $(03 \mathrm{H})$ revealed generally well-preserved morphology (Fig. 2c), although areas of degeneration and regeneration were detected infrequently. Supplementary Fig. 3 shows a large area of the tibialis cranialis of Valgus $(03 \mathrm{H})$ (Supplementary Fig. 3a, a'), better preserved than a corresponding area from Vampire (08A), a dog transplanted with autologous cells (Supplementary Fig. 3b, b'),
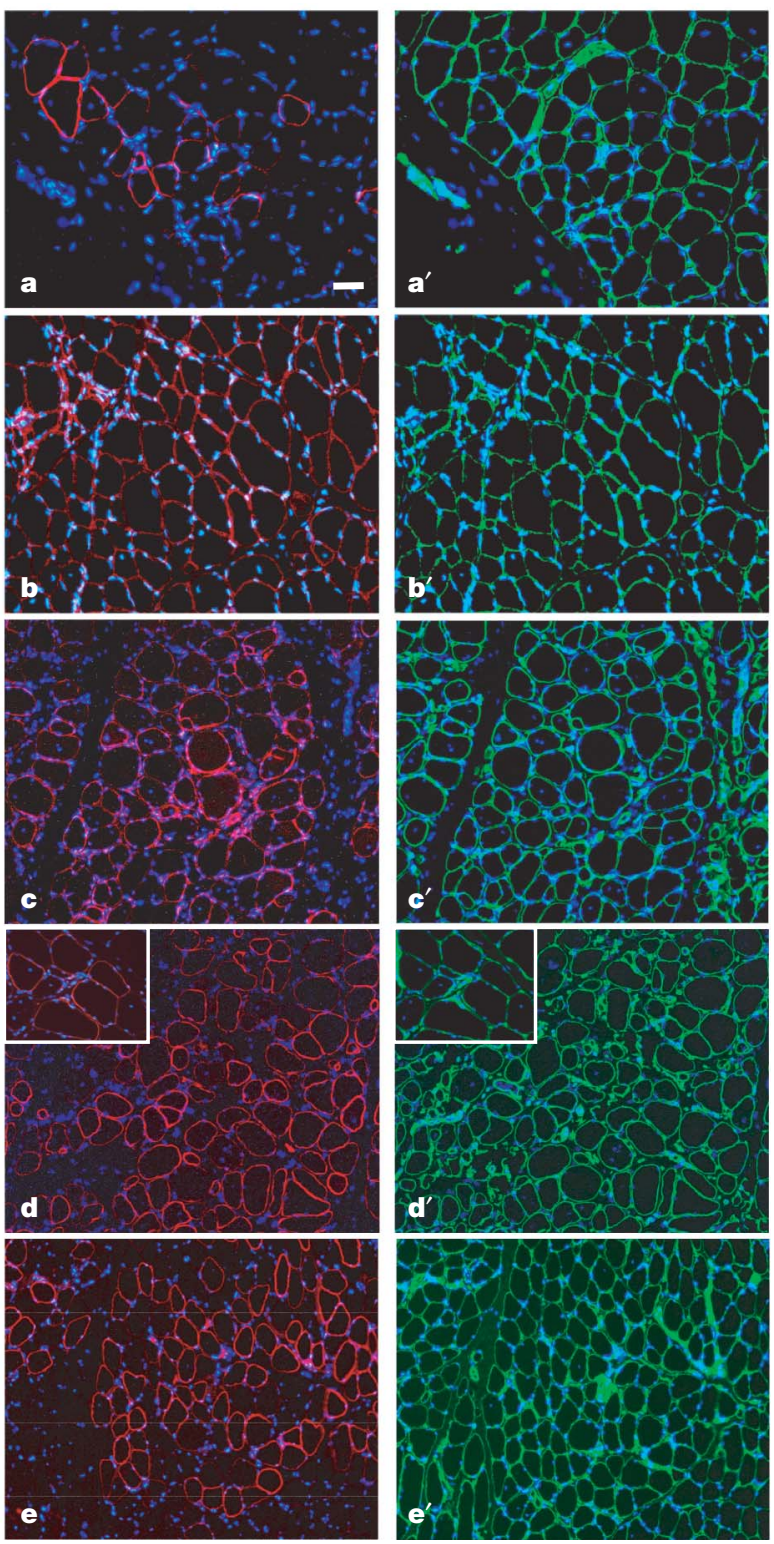

Figure 3 | Immunofluorescence analysis of tissue from treated dogs. Double immunofluorescence analysis of muscle biopsies from the tibialis cranialis of Ucal (01A; a, a'), Valgus $\left(03 \mathrm{H} ; \mathbf{b}, \mathbf{b}^{\prime}\right)$, Varus $\left(04 \mathrm{H} ; \mathbf{c}, \mathbf{c}^{\prime}\right)$, Vampire $\left(08 \mathrm{~A} ; \mathbf{d}, \mathbf{d}^{\prime}\right)$ and Azor $\left(10 \mathrm{H} ; \mathbf{e}, \mathbf{e}^{\prime}\right)$, stained with anti-laminin antibody (green in $\mathbf{a}^{\prime}-\mathbf{e}^{\prime}$ and inset in $\mathbf{d}^{\prime}$ ) and anti-dystrophin (red in a-d) or anti- $\beta$ sarcoglycan (red in inset in d). Nuclear staining with DAPI appears in blue. Note clusters of dystrophin-expressing fibres in Ucal, 01A (a) and extensive reconstitution of dystrophin-expressing fibres in Valgus $(03 \mathrm{H} ; \mathbf{b})$, Varus $(04 \mathrm{H} ; \mathrm{c})$ and Azor $(10 \mathrm{H} ; \mathrm{e})$. Several fibres expressing microdystrophin in Vampire, $08 \mathrm{H}(\mathrm{d})$ also express $\beta$-sarcoglycan in the same fibres stained on serial, non-adjacent sections (inset in d). Scale bar, $100 \mu \mathrm{m}$. 
described in detail below, which is itself better preserved than the biopsy from Akan (11U), an untreated dog (Supplementary Fig. 3c, $\left.c^{\prime}\right)$. Both forelimb muscles (not shown) and the diaphragm (Supplementary Fig. 5a) of Valgus $(03 \mathrm{H})$ also showed a relatively preserved morphology. Similarly, dystrophin was expressed in most of the examined biopsies from several different muscles of Valgus $(03 \mathrm{H} 9)$, such as tibialis cranialis (Fig. $\left.3 \mathrm{~b}, \mathrm{~b}^{\prime}\right)$, gastrocnemius and biceps femoralis (not shown). Large areas, containing several hundred dystrophin-expressing fibres (up to $70 \%$ of total fibres), were frequently detected: an example of the sartorius of Valgus $(03 \mathrm{H})$ is shown in Supplementary Fig. 5. Several clusters of dystrophin positive fibres could be detected even in the diaphragm (Supplementary Fig. $4 \mathrm{e})$.

The biopsies of Varus $(04 \mathrm{H})$ also showed well-preserved morphology although signs of muscle necrosis and regeneration were still present (Fig. 2d). Overall, most of the biopsies in all muscles were morphologically less affected than those of untreated dogs. Dystrophin expression in these biopsies was variable but large areas of dystrophin-positive fibres could be detected, as shown in Fig. 3c, $c^{\prime}$. In addition, the diaphragm of Varus was partly preserved (Supplementary Fig. 4b) and contained clusters of dystrophin-positive fibres (Supplementary Fig. 4f). Figure 4 shows the percentage of dystrophin-expressing fibres, which ranged from $10 \%$ to at least $70 \%$ in two distant sections of three different biopsies each of selected muscles from Valgus $(03 \mathrm{H})$ and Varus $(04 \mathrm{H})$. Western blot analyses of extracts from different biopsies of the same muscles confirmed the presence of significant amounts of dystrophin, varying from an undetectable signal to about $60 \%$ of a control wild-type canine muscle (Fig. 4c). When dystrophin was clearly detected, $\beta$-sarcoglycan was also detected, indicating reconstitution of the dystrophin-associated complex.

\section{Efficacy of autologous mesoangioblasts}

Three dogs, Vaccin, Valium and Vampire (06A, 07A and 08A, respectively), received their own mesoangioblasts, transduced with the new lentiviral vector expressing human microdystrophin. Like Ucal (01A) in the first experiment, they received the first injection at about 4 months of age (P113). Valium (07A) and Vampire (08A) died of pneumonia after the third and fifth injection, respectively. Because of early death, no force measurement was performed but a movie showing reduced walking ability in Vampire (08A) was taken few days before his death (Supplementary Movie 4) and biopsies were taken at autopsy. Valium (07A) received systemic arterial delivery, like Valgus $(03 \mathrm{H})$, and maintained a certain walking ability, albeit very stiff. The biopsies of the three dogs revealed partly preserved morphology; for example, tibialis cranialis from Vaccin (06A) is shown in Fig. 2d. In addition, microdystrophin expression in the tibialis cranialis of Vampire (08A) was significantly widespread (Fig. 3d, d'), despite the poor clinical performance. The microdystrophin was able to recruit at least some member of the dystrophin-associated complex such as $\beta$-sarcoglycan (shown in a serial, non-adjacent section in insets to Fig. $3 \mathrm{~d}, \mathrm{~d}^{\prime}$ and by western blot analysis in Fig. 4b). A quantitative analysis of microdystrophin for Vaccin (06A) and Vampire (08A) is reported in Fig. 4. Thus, all three dogs treated with autologous, genetically corrected cells performed poorly, even though two of them showed amelioration of morphology and microdystrophin-expressing fibres, ranging from 5\% to $50 \%$.

\section{Efficacy of late transplantation of donor mesoangioblasts}

To verify whether the less effective results obtained with autologous cells were due to the later onset of the treatment, two dogs, Azur $(09 \mathrm{H})$ and Azor $(10 \mathrm{H})$, received the first of five injections at P159 (5 months), 1 month later than those animals treated with their own genetically modified cells. Although both dogs were already severely affected at the onset of treatment, Azor $(10 \mathrm{H})$ showed a striking improvement of motility (while still limping) and was even able to run at the end of treatment (Supplementary Movie 5). Azur (09H) showed a less evident but clearly detectable amelioration (Supplementary Movie 6). In particular, although there was variation between dogs in the progression of the disease, spontaneous improvement was never observed. Biopsies taken at the end of treatment also showed relatively well-preserved morphology (although with sclerosis and infiltrates) and widespread dystrophin expression in the tibialis cranialis (Fig. 4e, e' ), biceps femoralis, gastrocnemius, sartorius and even diaphragm (Supplementary Fig. 6 and data not shown). Thus, we conclude that even with a later onset of treatment, donor cells seem to produce a greater amelioration of muscular dystrophy than is produced by autologous microdystrophin-expressing cells. After the end of treatment with cyclosporine, Azor $(10 \mathrm{H})$ continued to walk actively until the end of the experiment, whereas Azur $(09 \mathrm{H})$ rapidly lost walking ability, much as had previously been observed with Valgus $(03 \mathrm{H})$ and Varus $(04 \mathrm{H})$.
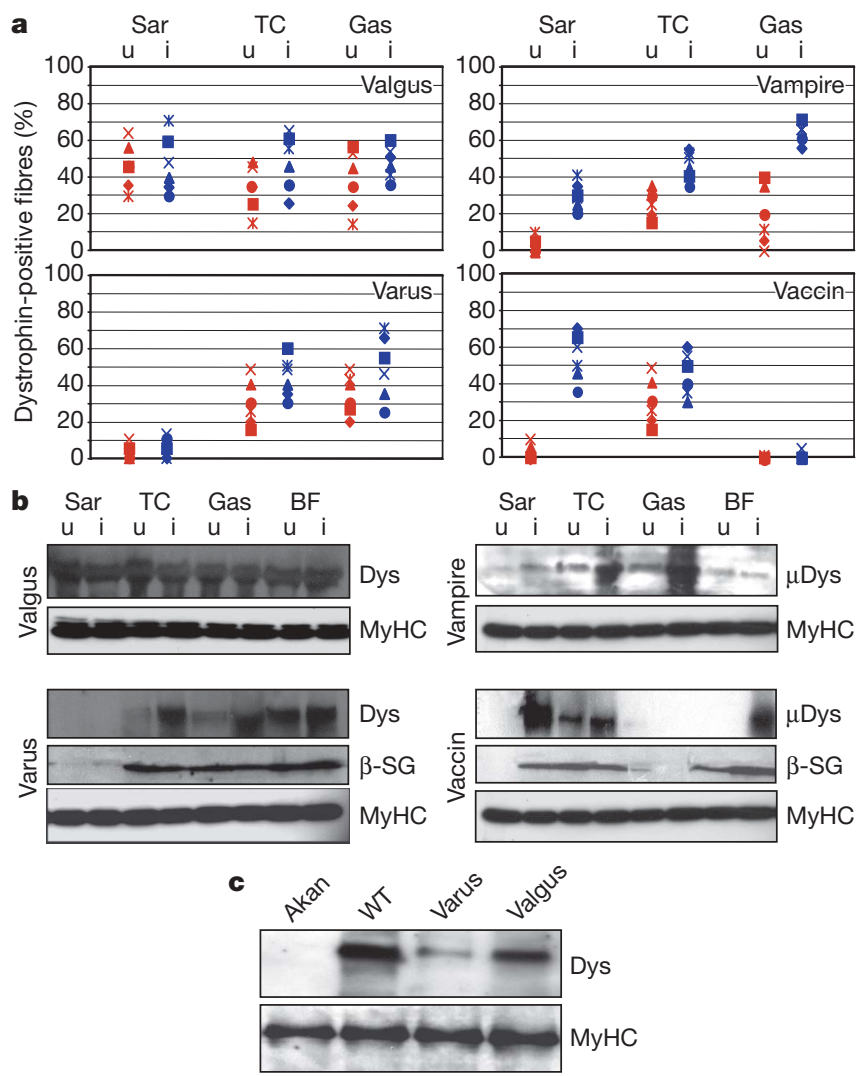

Figure 4 | Quantitative analysis of dystrophin content in tissue from treated dogs. a, Three separate sections for two different biopsies of the injected (i, blue symbols) and non-injected (u, red symbols) sartorius (Sar), tibialis cranialis (TC) and gastrocnemius (Gas) for two dogs injected with donor cells (Valgus $(03 \mathrm{H})$ and Varus $(04 \mathrm{H})$ ) and two dogs injected with autologous, genetically corrected, cells (Vaccin (06A) and Vampire (08A)) were analysed for dystrophin expression. A total of 200 laminin-positive fibres (of any size) were counted in randomly selected fields, and the percentage of these fibres also expressing dystrophin was calculated. b, Western blot analysis of different biopsies from the muscles shown in a plus the biceps femoralis (BF) showing the expression of dystrophin (Dys, Valgus $(03 \mathrm{H})$ and Varus $(04 \mathrm{H}))$ or microdystrophin $(\mu \mathrm{Dys}$, Vaccin $(06 \mathrm{~A})$ and Vampire (08A)). Each lane was loaded with $80 \mu \mathrm{g}$ of proteins. Myosin heavy chains (MyHC) are also shown as an internal standard in the bottom panel. For Varus $(03 \mathrm{H})$ and Vampire $(08 \mathrm{~A})$ the expression of $\beta$-sarcoglycan ( $\beta$-SG) is also shown in the middle panel. There is a good correlation between the expression of dystrophin or microdystrophin and $\beta$-sarcoglycan. c, Expression of dystrophin and myosin heavy chains in the injected biceps femoralis of GMRD untreated (Akan (U11)), wild type, Varus $(04 \mathrm{H})$ and Valgus $(03 \mathrm{H})$; only $30 \mu \mathrm{g}$ of total proteins was loaded in each lane for direct comparison. 


\section{Analysis of muscle-released serum enzymes}

Throughout treatment, serum collected from treated dogs was used to detect the levels of creatine kinase and other enzymes released by damaged muscle fibres (Supplementary Fig. 7); in general, the injection of mesoangioblasts corresponded to rapid and often profound decrease in enzyme activity. This may be due to improved survival of fibres reconstituted with transplanted cells but also to factors such as insulin-like growth factor 1, basic fibroblast growth factor and others released by donor cells ${ }^{15}$ and contributing to fibre survival. At the end of treatment, the level of creatine kinase in all treated dogs was well below the those reported for this form of muscular dystrophy ${ }^{9}$. Other enzymes released by damaged muscle fibres, such as aspartate aminotransferase, showed an overall similar pattern (data not shown).

\section{Enhancement of contraction force}

To test whether morphological and biochemical changes would correspond to increased force of contraction we performed two different types of analysis: tetanic force of the tibialis cranialis and extensor digitorum longus muscle for Valgus $(03 \mathrm{H})$, Varus $(04 \mathrm{H})$,
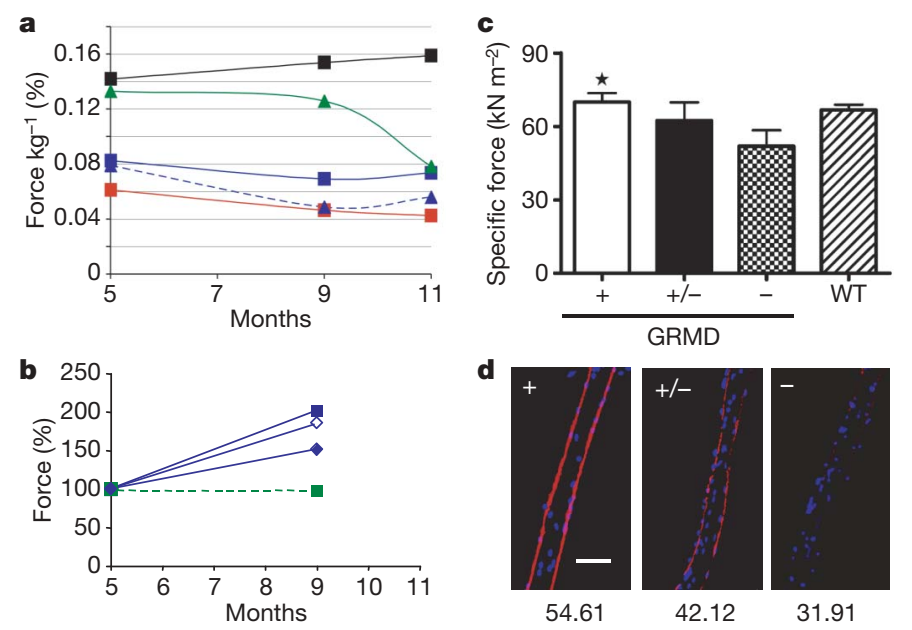

Figure 5 | Physiology. Functional properties of skeletal muscles in vivo and of individual muscle fibres in vitro of treated dystrophic dogs after five consecutive injections of donor wild-type mesoangioblasts. a, Tetanic force of the flexor muscles of the cranial tibial compartment of different dogs measured after maximal stimulation of the common peroneal nerve and recorded with an ergometer developed in house. Black squares, normal wildtype dog; red squares, dystrophic untreated dog (Akan (11U)); green triangles: dystrophic dog transplanted with autologous genetically corrected mesoangioblasts (Vaccin (06A)); blue symbols, dystrophic dogs transplanted with donor wild-type mesoangioblasts (squares, Valgus $(03 \mathrm{H})$ and Varus $(04 \mathrm{H})$; triangles, Azur $(09 \mathrm{H})$ and Azor $(10 \mathrm{H}))$. Tetanic force was normalized to each dog's body weight to obtain weight-corrected specific force. $\mathbf{b}$, Relative increase in force of the flexor muscles of the cranial tibial compartment of the injected leg versus the untreated leg. Blue squares, Varus (04H); green squares, Vaccin (06A); filled blue diamonds, Azur (09H); open blue diamonds, Azor $(10 \mathrm{H})$. c, Specific force (maximum isometric force/cross-sectional area) of a population of 199 single muscle fibres dissected from tibialis cranialis, gastrocnemius, and sartorius muscles of Valgus $(03 \mathrm{H})$ and Varus $(04 \mathrm{H})$, indicated together as GRMD, and of 148 fibres dissected from the same muscles of two control golden retriever dogs (WT). All fibres shown are fast fibres (type 2A, 2AX or $2 \mathrm{X})(n=71$ for GRMD; $n=106$ for WT). Type 2A, 2AX and 2X fibres were pooled together because no difference in specific force was observed between these fast fibre types in either GRMD or WT dogs. Fibres from Valgus $(03 \mathrm{H})$ and Varus $(04 \mathrm{H})$ were grouped into dystrophin-positive $(+)$, partly positive $(+/-)$ and negative $(-)$ on the basis of the presence of dystrophin detected, after force determination, by immunostaining by anti-dystrophin antibody.

d, Examples of positive $(+)$, partly positive $(+/-)$ and negative $(-)$ single skinned fibres immunostained for dystrophin after force determination. The specific force (maximum isometric force/cross sectional area) developed by each fibre is shown under each panel. Scale bar, $100 \mu \mathrm{m}$. Error bars, 1 s.d.
Vaccin (06A), Azur $(09 \mathrm{H})$ and Azor $(10 \mathrm{H})$, and force of contraction on isolated single fibres from Varus $(03 \mathrm{H})$, Valgus $(04 \mathrm{H})$, Vaccin (06A) and Valium (07A). Figure 5a shows the tetanic force of a normal dog (black squares), which increases progressively with age. Dystrophic dogs have variable tetanic force (ranging from $90 \%$ to $40 \%$ of wild type) but, in the absence of treatment (red squares), progressively lose it. In the experiment reported, Vaccin (06A), who received autologous cells (green triangles), showed a strong tetanic force at the onset of treatment and maintained it up to the fourth injection but later showed a significant decrease. In contrast, the four dogs receiving donor cells (blue squares and triangles) started from a decreased tetanic force but maintained it though the treatment and eventually showed a modest increase in the last measurement, at the end of treatment. To avoid the problems deriving from this variability, force was also measured and reported as the percentage increase in treated over untreated legs. Figure $5 \mathrm{~b}$ shows that all dogs receiving donor cells showed an increase in tetanic force ranging from $50 \%$ to $100 \%$ in the treated leg, whereas the dog receiving his own cells (Vaccin, 06A) did not show any increase. To measure force of contraction at the singlefibre level, a large population of single skinned fibres was dissected from the tibialis cranialis, gastrocnemius and sartorius muscles of Valgus $(03 \mathrm{H})$ and Varus $(04 \mathrm{H})$ and of two control wild-type dogs. After determination of specific force, each fibre was also analysed for the expression of dystrophin and of fast or slow myosin heavy chains (MyHC). The results reported in Fig. 5c show that fast fibres from dystrophic dogs had a complete recovery in force when expressing dystrophin, up to the level of fibres from wild-type dogs. Fibres showing partial expression of dystrophin also showed partial force recovery. A picture showing fully or partly reconstituted as well as dystrophin-negative fibres is shown in Fig. 5d. In contrast, dystrophic slow fibres did not show reduced force in comparison with wild-type fibres, as shown previously ${ }^{16}$ (data not shown). A similar measurement on the single skinned fibres of the tibialis cranialis and sartorius of Vaccin and Valium (06A and (07A) showed a similar trend, but the increase in force of contraction was modest and not statistically significant (data not shown).

\section{Immune reaction against dystrophin and donor cells}

To test the possible occurrence of an immune reaction in the transplanted dogs against donor cells and/or the transgene (dog dystrophin and human microdystrophin, respectively), we performed an immunocytochemical analysis of cellular infiltrates, a western blot analysis to test the reactivity of dog sera and a lymphocyte proliferation assay to test the appearance of cellular immunity against donor cells and/or the transplanted muscle tissue ${ }^{17-19}$. Results indicate a low frequency of infiltrates, an absence of serum antibodies and modest activation only for local lymphocytes exposed to transplanted muscle tissue (Supplementary Information).

\section{Conclusions and future perspectives}

Gene or cell therapy approaches for GRMD have until now produced negative $e^{20}$ or modestly positive results ${ }^{21-23}$. We show here that it is possible to transplant mesoangioblasts into dystrophic dogs and obtain an extensive reconstitution of fibres expressing dystrophin, an improvement in the contraction force and, in many cases, a preservation of walking ability. Previous work in the mouse ${ }^{4,13}$ showed that mesoangioblasts express some of the protein that leukocytes use to cross the vessel wall and so they invade the interstitial tissue, eventually to fuse with and contribute sarcoglycan to regenerating fibres. Donor wild-type mesoangioblasts seemed to be more efficient than autologous, genetically corrected cells. Possibly microdystrophin produces a modest functional rescue when delivered late through donor cells in contrast with the excellent functional rescue induced when delivered as a transgene ${ }^{11}$. A different onset of treatment should not be crucial because two late-transplanted dogs (Azor 
$(09 \mathrm{H})$ and Azur $(10 \mathrm{H}))$ showed improved motility; in addition, the differential treatment with cyclosporine should not matter because the drug itself had no effect on dogs transplanted with wild-type bone marrow $^{19}$ (S.B. and C. Escriou, unpublished observation).

As mentioned above, extensive variability exists between dystrophic dogs; this is the situation that will be faced with patients, who also show phenotypic variability within the same mutation ${ }^{24}$. Even taking this into account, four of six dogs treated with donor cells showed clear clinical amelioration; of the remaining two, Viko $(05 \mathrm{H})$ died early and could not be analysed in detail and Vrillie $(02 \mathrm{H})$ probably received too few cells. At the end of immune suppression, two of the four dogs showing amelioration, namely Valgus $(03 \mathrm{H})$ and Azor $(10 \mathrm{H})$, continued to walk well until the end of the experiment, whereas the other two, Varus $(04 \mathrm{H})$ and Azur $(09 \mathrm{H})$, rapidly lost walking ability. We do not have an explanation for this difference, which may reflect the different survival of transplanted organs after the end of immune suppression ${ }^{25}$.

Extrapolation of these results to a possible future clinical trial would suggest starting with donor cells from an HLA-matched donor under a regime of immune suppression. The absence of cells of the immune system in the donor population should create a more favourable situation than with bone marrow transplantation. In fact, the modest immune reaction detected against donor canine mesoangioblasts, which were not DLA matched, indicates that immune suppression might be modest and perhaps transient.

Thus, the work reported here sets the logical premise for the start of clinical experimentation that may lead to an efficacious therapy for Duchenne muscular dystrophy.

\section{METHODS}

Cells and culture conditions. Dog mesoangioblasts were isolated from muscle biopsies of golden retriever dystrophic dogs (GRMD) and wild-type golden retrievers as described previously ${ }^{4}$. Details are given in Supplementary Information.

In vivo cell migration assay. Dog mesoangioblasts were analysed for the ability to migrate in vivo as described ${ }^{13}$. Details are given in Supplementary Information. Fluorescence-activated cell sorting. For fluorescence-activated cell sorting, cells were detached and stained sequentially with primary antibodies against CD13, CD31, CD34, CD44, CD 117 and CD45 (Becton Dickinson) and immunofluorescent secondary antibodies and fixed with $2 \%$ paraformaldehyde until analysis with a FACSCalibur (Becton Dickinson).

Intra-artery delivery of mesoangioblasts in GRMD. About $5 \times 10^{7}$ mesoangioblasts were injected through the femoral artery of ten dogs, as detailed in Supplementary Information.

Biochemical and histological analysis. Immunoblotting, immunofluorescence and histological staining were performed as previously described ${ }^{4}$ and detailed in Supplementary Information.

In vivo analysis of muscle force. Maximal isometric force generated by flexor muscles of the tibialis cranialis compartment (tibialis cranialis and extensor digitorum longus) was measured by supramaximal percutaneous stimulation of the common peroneal nerve with a set-up detailed in Supplementary Information.

Clinical GRMD follow-up. Male golden retrievers carrying the GRMD mutation obtained from a colony at the veterinary school of Alfort were observed for disease for at least 1 month before being entered into the study. Details are given in Supplementary Information.

Received 4 April; accepted 26 September 2006.

Published online 15 November 2006.

1. Emery, A. E. The muscular dystrophies. Lancet 359, 687-695 (2002)

2. Cossu, G. \& Sampaolesi, M. New therapies for muscular dystrophy: cautious optimism. Trends Mol. Med. 10, 516-520 (2004)

3. Qu-Petersen, Z. et al. Identification of a novel population of muscle stem cells in mice: potential for muscle regeneration. J. Cell Biol. 157, 851-864 (2002).

4. Sampaolesi, M. et al. Cell therapy of $\alpha$-sarcoglycan null dystrophic mice through intra-arterial delivery of mesoangioblasts. Science 301, 487-492 (2003).

5. Bachrach, E. et al. Systemic delivery of human microdystrophin to regenerating mouse dystrophic muscle by muscle progenitor cells. Proc. Natl Acad. Sci. USA 101, 3581-3586 (2004)
6. Torrente, Y. et al. Human circulating $\mathrm{AC} 133^{+}$stem cells restore dystrophin expression and ameliorate function in dystrophic skeletal muscle. J. Clin. Invest. 114, 182-195 (2004).

7. Rodriguez, A. M. et al. Transplantation of a multipotent cell population from human adipose tissue induces dystrophin expression in the immunocompetent mdx mouse. J. Exp. Med. 201, 1397-1405 (2005).

8. Dezawa, M. et al. Bone marrow stromal cells generate muscle cells and repair muscle degeneration. Science 309, 314-317 (2005).

9. Kornegay, J. N., Tuler, S. M., Miller, D. M. \& Levesque, D. C. Muscular dystrophy in a litter of golden retriever dogs. Muscle Nerve 11, 1056-1064 (1988).

10. Sharp, N. J. et al. An error in dystrophin mRNA processing in golden retriever muscular dystrophy, an animal homologue of Duchenne muscular dystrophy. Genomics 13, 115-121 (1992).

11. Harper, S. Q. et al. Modular flexibility of dystrophin: implication for gene therapy of Duchenne muscular dystrophy. Nature Med. 8, 253-261 (2004).

12. Minasi, M. G. et al. The meso-angioblast: a multipotent, self-renewing cell that originates from the dorsal aorta and differentiates into most mesodermal tissues. Development 129, 2773-2783 (2002).

13. Galvez, B. G. et al. Complete rescue of dystrophic muscle by mesoangioblasts with enhanced migratory ability. J. Cell Biol. 174, 231-243 (2006).

14. Kornegay, J. N., Cundiff, D. D., Bogan, D. J., Bogan, J. R. \& Okamura, C. S. The cranial sartorius muscle undergoes true hypertrophy in dogs with golden retriever muscular dystrophy. Neuromuscul. Disord. 13, 493-500 (2003).

15. Galli, D. et al. Mesoangioblasts, vessel-associated multipotent stem cells, repair the infarcted heart by multiple cellular mechanisms: a comparison with bone marrow progenitors, fibroblasts, and endothelial cells. Arterioscler. Thromb. Vasc. Biol. 25, 692-697 (2005).

16. Childers, M. K. et al. Skinned single fibers from normal and dystrophin-deficient dogs incur comparable stretch-induced force deficits. Muscle Nerve 31, 768-771 (2005).

17. Pavlath, G. K. Regulation of class I MHC expression in skeletal muscle: deleterious effect of aberrant expression on myogenesis. J. Neuroimmunol. 125, 42-50 (2002).

18. Krampera, M. et al. Bone marrow mesenchymal stem cells inhibit the response of naive and memory antigen-specific T cells to their cognate peptide. Blood 101, 3722-3729 (2003)

19. Reinhardt, R. L. et al. Visualizing the generation of memory CD4 T cells in the whole body. Nature 410, 101-105 (2001)

20. Dell'Agnola, C. et al. Hematopoietic stem cell transplantation does not restore dystrophin expression in Duchenne muscular dystrophy dogs. Blood 104, 4311-4318 (2004)

21. Karpati, G., Gilbert, R., Petrof, B. J. \& Nalbantoglu, J. Gene therapy research for Duchenne and Becker muscular dystrophies. Curr. Opin. Neurol. 10, 430-435 (1997).

22. Howell, J. M. et al. Use of the dog model for Duchenne muscular dystrophy in gene therapy trials. Neuromuscul. Disord. 7, 325-328 (1997).

23. Cerletti, M. et al. Dystrophic phenotype of canine X-linked muscular dystrophy is mitigated by adenovirus-mediated utrophin gene transfer. Gene Ther. 10, 750-757 (2003).

24. Emery, A. E. Clinical and molecular studies in Duchenne muscular dystrophy. Prog. Clin. Biol. Res. 306, 15-28 (1989).

25. Ricordi, C.\& Strom, T. M. Clinical islet transplantation: advances and clinical challenges. Nature Rev. Immunol. 4, 259-270 (2004).

Supplementary Information is linked to the online version of the paper at www.nature.com/nature.

Acknowledgements We thank M. G. Roncarolo for helpful discussions; J. Chamberlain for the gift of the lentiviral vector expressing human microdystrophin; C. Drougard for technical assistance; X. Cauchois, I. Gruyer, S. Kouamé, E. Wembe and A. Brindejont and M. Carré at the Centre d'Elevage du Domaine des Souches for their care of the dogs; and N. Borenstein for the systemic delivery of cells. M.S. and G.C. thank P. Luban for a donation. This work was supported by grants from the Association Française contre les Myopathies, Telethon, the Muscular Dystrophy Association, the Parent Project Onlus, the European Community 'Eurostemcell', the Cariplo Foundation and the Italian Ministries of Health and Research. B.G.G. was supported by a $3+3$ fellowship from the Centro Nacional de Investigationes Cardiovasculares, Spain.

Author Contributions M.S. coordinated the work on cells with R.T. and M.G.C.D.; S.B. coordinated the work on dogs with N.G., J.L.T. and I.B.; R.B. and G.D.A. coordinated the physiology experiments with O.P., C.R. and P.M., who also developed, with S.M., the instrument to measure dog tetanic force; A.I. did the histology work; B.G.G. performed the homing experiments; L.P. and M.S. conducted the western blot analysis; M.G. did the immunology experiment; Y.T. and C.B. evaluated the clinical aspects of the work; G.C. coordinated the whole project.

Author Information Reprints and permissions information is available at www.nature.com/reprints. The authors declare no competing financial interests. Correspondence and requests for materials should be addressed to C.G. (cossu.giulio@hsr.it) or R.B. (roberto.bottinelli@unipv.it). 